\title{
Time Course of the Suppression Effect on Transient Evoked Otoacoustic Emissions by Prolonged Contralateral Acoustic Stimulation
}

\author{
Hyun Woo Kang, Hyun Joon Shim, Seong Jun Song, Seong Hee Lee and Sang Won Yoon \\ Department of Otolaryngology, Eulji Medical Center, Eulji University School of Medicine, Seoul, Korea
}

Received September 25, 2012

Revised November 29, 2012

Accepted December 5, 2012

Address for correspondence

Hyun Joon Shim, MD

Department of Otolaryngology,

Eulji Medical Center,

Eulji University School of Medicine,

Hangeulbiseok-ro 14-gil, Nowon-gu,

Seoul 139-872, Korea

Tel $+82-2-970-8276$

Fax $+82-2-970-8275$

E-mail eardoc11@naver.com
Background and Objectives: Although the suppressive effect of the medial efferent acoustic reflex is well known, the time course of this effect over prolonged periods has yet to be fully evaluated. We assessed time-dependent change in the suppression of transient evoked otoacoustic emissions (TEOAEs) by the medial efferent acoustic reflex over a relatively long period. Subjects and Methods: We measured TEOAEs in the right ear before contralateral acoustic stimulation (CAS), and then measured serial TEOAEs in the right ear at four intervals during a total of 16 minutes of continuous CAS, followed by three more recordings after termination of CAS. Results: TEOAE amplitudes were reduced with CAS during a certain period (from the immediate period to 10 minutes depending on frequency) and subsequently recovered. TEO$\mathrm{AE}$ suppression values in the mean amplitudes for overall frequency were $0.76 \mathrm{~dB}$ at the initial recording, $0.35 \mathrm{~dB}$ at 5 minutes, $0.44 \mathrm{~dB}$ at 10 minutes, and $0.33 \mathrm{~dB}$ at 15 minutes during CAS The initial suppression value was significantly larger than other suppression values of 5,10 , and 15 minutes $(p<0.05)$. In recordings obtained after CAS, TEOAE amplitude exceeded preacoustic amplitudes at $1 \mathrm{kHz}, 1.5 \mathrm{kHz}$, and $2 \mathrm{kHz}$. Conclusions: The present results show the existence of the medial efferent acoustic reflex and demonstrate the time course that TEOAE suppressions present initially after CAS, showing fatigue over time. Overshooting of TEOAE was observed in recordings at several frequencies after termination of CAS.

Korean J Audiol 2012;16:114-119

KEY WORDS: Otoacoustic emissions · Acoustic reflex · Efferent fiber · Acoustic stimulation

\section{Introduction}

Otoacoustic emissions (OAEs) have become a useful, noninvasive, objective measurement of outer hair cell response since their discovery by Kemp ${ }^{1)}$ in 1978. OAEs are generated by the outer hair cells of the cochlea and can be recorded in the external auditory canal. OAEs are created by an outer hair cell active amplification mechanism, and these sounds can be reduced by contralateral noise. The medial olivocochlear bundle efferent pathway is composed of ipsilateral and contralateral neurons. Acoustic stimulation of a single side stimulates type I afferent fibers of the auditory nerve. These fibers project centrally to the cochlear nucleus, where they synapse. Reflex interneurons innervate the ipsilateral and contralateral superior olivary complex. The contralateral outer hair cells are then innervated by the bilateral medial olivocochlear fiber bundle to suppress the mechanical response of the outer hair cells. ${ }^{2,3)}$ The medial olivocochlear bundle acts to protect the cochlea from acoustic overstimulation. ${ }^{4)}$ Another possible function of the medial olivocochlear system is in facilitating selective attention. The central nervous system uses the medial efferent pathway to cause the outer hair cells to change the micromechanical properties of the cochlea during selective attention tasks. ${ }^{3,5)}$

Previous attempts to study the action duration and characteristics of the efferent effect of the medial olivocochlear system in animals and humans using different recording methods have delivered inconsistent results. Some studies showed that suppression reached a constant steady state during contralateral acoustic stimulation (CAS), ${ }^{6,7)}$ whereas another reported a gradual release of suppression during $\mathrm{CAS}$. $^{8)}$ In addition, when contralateral acoustic stimulation was terminated, some research- 
ers reported that amplitude exceeded the corresponding control amplitude, showing significant enhancement and overshoot, ${ }^{9)}$ while other researchers reported residual suppression of amplitude after sound termination. ${ }^{7)}$

The aim of the present study was to evaluate the time course of the efferent effect of the medial olivocochlear system in humans in terms of the amplitude of transient evoked OAEs (TEOAEs), using sufficiently prolonged CAS.

\section{Subjects and Methods}

We used an experimental setting similar to that described by van Zyl, et al., ${ }^{9)}$ but with a larger study cohort.

\section{Participants}

Twenty adults ( 5 men, 15 women; age range, 23-35 years; mean age, $26.9 \pm 5.3$ years) with normal hearing abilities were evaluated in this study. All subjects met the following criteria based on a subjective clinical interview: 1) no history of past otologic disease, 2) no familial history of hearing loss, and 3) no history of ototoxic drug use. All subjects presented with normal appearance of the tympanic membrane and external auditory canal. All subjects underwent audiologic testing, which revealed that all had hearing thresholds less than 20 $\mathrm{dB}$ between 250 and $8000 \mathrm{~Hz}$, and had normal type A tympanogram and normal stapedial reflex at $90 \mathrm{~dB}$ HL.

\section{Procedure}

TEOAEs were recorded and analyzed using the Scout Sport system in Navigator pro (Bio-logic Systems Corp., Chicago, IL, USA) in a sound-treated audiological test booth. Linear broadband $80 \mu$ s clicks were presented at $80 \mathrm{~dB}$ peak equivalent SPL at a repetition rate of $40 / \mathrm{s}$ and recorded in the right ear of each subject. TEOAEs were evaluated at frequency bands of 1000, 1500, 2000, 3000, and $4000 \mathrm{~Hz}$, and 260 times averages were completed to reduce the noise effect. The average duration of a TEOAE recording was 1 minute. CAS was presented through earphones at an intensity of $45 \mathrm{~dB}$ SL to the left ear of each subject. CAS was white noise (duration, $300 \mathrm{msec}$; rise-fall time, $0.5 \mathrm{~ms}$; sampling, $44.1 \mathrm{kHz}$ ). TEOAE recordings were measured under experimental conditions without CAS, and then at 5-minute intervals over a period of 16 minutes with CAS. After 16 minutes of CAS, TEOAEs were recorded at 1,3 , and 5 minutes without any acoustic stimulation. Over the course of the experiments, TEOAEs were measured eight times in each subject (Fig. 1).

The mean TEOAE amplitudes of all subjects were compared between pre-acoustic stimulation and four times during acoustic stimulation at $1,1.5,2,3$, and $4 \mathrm{kHz}$ and the mean TEOAE amplitudes of all subjects at 1, 1.5, 2, 3, and $4 \mathrm{kHz}$ were compared between pre-acoustic stimulation and after termination of acoustic stimulation. Suppression values (difference between pre-acoustic stimulation amplitude and amplitude during acoustic stimulation) and enhancement values (difference between pre-acoustic stimulation amplitude and amplitude after acoustic stimulation) were calculated for the mean amplitudes of overall frequency, and suppression values were compared as a function of CAS duration.

\section{Statistical analysis}

Paired t-test was used to compare TEOAE amplitudes between pre-acoustic stimulation and four times during acoustic stimulation, and between pre-acoustic stimulation and postacoustic stimulation, using SPSS software. Repeated ANOVA was applied to compare suppression values for overall frequency as a function of CAS duration and to compare suppression values among the different frequencies.

\section{Results}

A significant reduction in the mean amplitude of the $1 \mathrm{kHz}$ TEOAEs by acoustic stimulation was detected initially, at 5
Fig. 1. Schematic drawing of the study procedure. TEOAEs were performed in the right ear before contralateral acoustic stimulation and 4 serial TEOAEs were measured in the right ear at 5-minute intervals during a total 16 minutes of contralateral acoustic stimulation. Three more serial TEOAEs were conducted at 2minute intervals, starting 1 minute after termination of the contralateral acoustic stimulation. A total of $8 \mathrm{se}-$ rial TEOAEs were performed. TEOAEs: transient evoked otoacoustic emissions, CAS: contralateral acoustic stimulation.

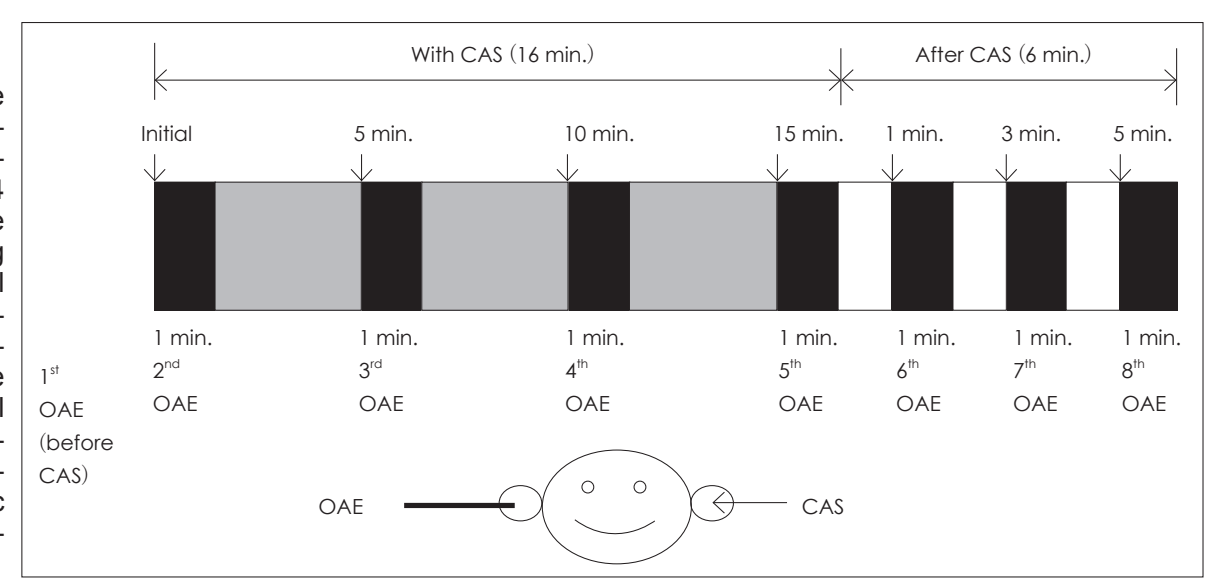




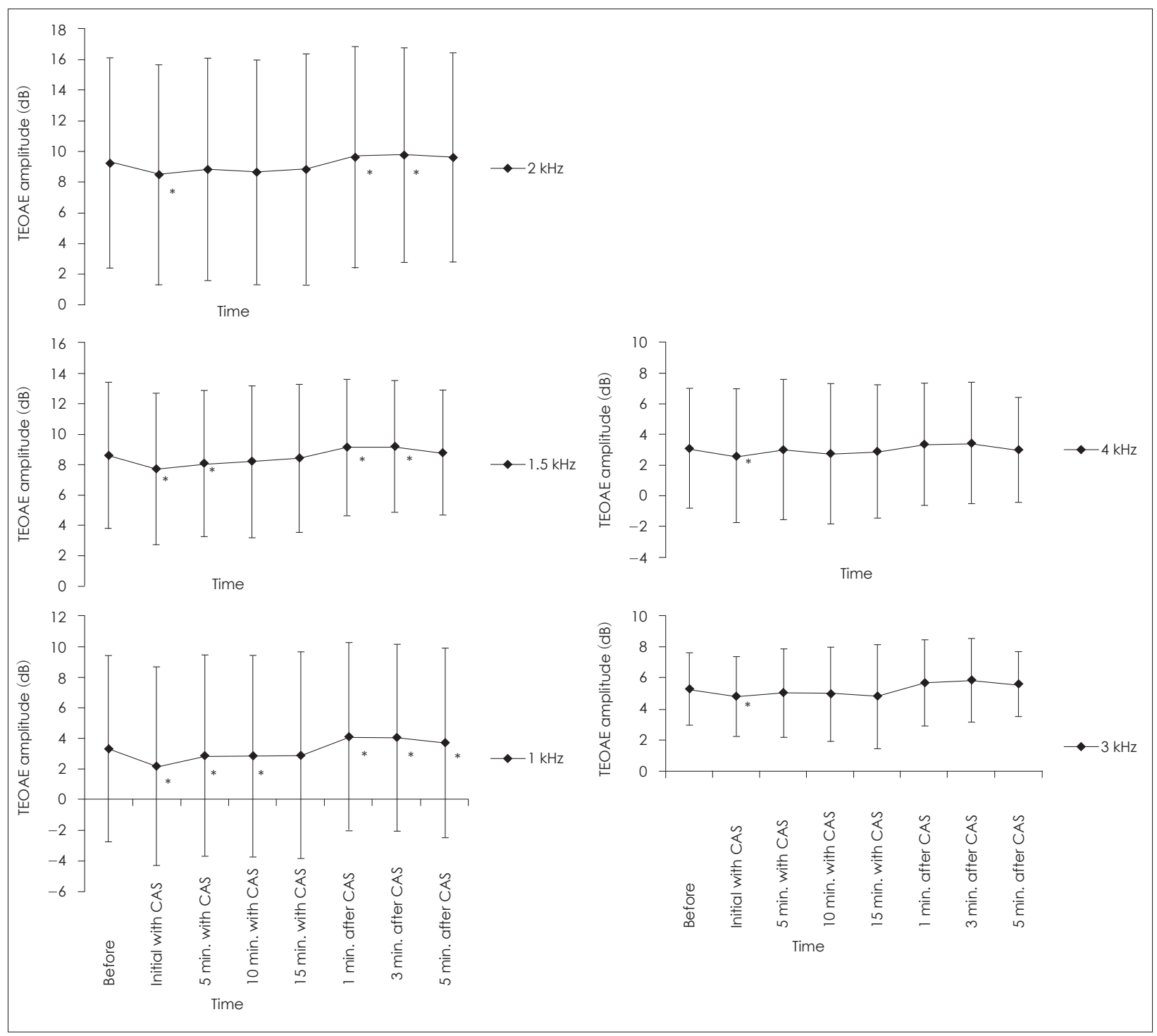

Fig. 2. Mean TEOAE amplitudes at 1, 1.5, 2, 3, and $4 \mathrm{kHz}$ obtained a total of 8 times throughout the study period. Significant differences in amplitudes between pre-acoustic stimulation and after acoustic stimulation are indicated by asterisks $\left({ }^{*}<0.05\right)$. TEOAE: transient evoked otoacoustic emission, CAS: contralateral acoustic stimulation.

minutes, and at 10 minutes after acoustic stimulation; in contrast, recorded values after 15 minutes showed no significant reduction. Recorded values at 1, 3, and 5 minutes after termination of acoustic stimulation showed a significant increase compared with the amplitudes recorded for pre-acoustic stimulation at $1 \mathrm{kHz}$ (Fig. 2).

At $1.5 \mathrm{kHz}$, a significant reduction was present initially and at 5 minutes after acoustic stimulation; the recorded values at 1 and 3 minutes after termination of acoustic stimulation showed a significant increase compared with the amplitudes recorded for pre-acoustic stimulation (Fig. 2). At $2 \mathrm{kHz}$, a significant reduction was detected only for initial recordings with acoustic stimulation; the recorded values at 1 and 3 minutes after termination of acoustic stimulation showed a signif- icant increase (Fig. 2). At $3 \mathrm{kHz}$, a significant reduction in the mean amplitude was present only for initial recordings with acoustic stimulation; there was no other significant difference in amplitude at any point when comparing pre-acoustic stimulation and after termination of acoustic stimulation (Fig. 2). As was found at frequencies of $3 \mathrm{kHz}$, acoustic stimulation at $4 \mathrm{kHz}$ showed a significant reduction in the mean amplitude only in the initial recordings, and a significant increase was not shown at any recording after termination of acoustic stimulation (Fig. 2).

Suppression values in the mean amplitudes for overall frequency were $0.76 \mathrm{~dB}$ (12.82\% suppression from pre-acoustic stimulation) at the initial recording, $0.35 \mathrm{~dB}$ (5.90\% suppression) at 5 minutes, $0.44 \mathrm{~dB}(7.42 \%$ suppression $)$ at 10 minutes, 


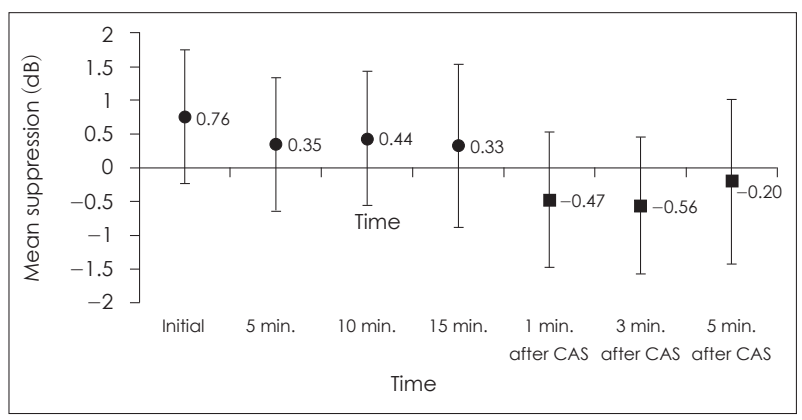

Fig. 3. Mean TEOAE suppression values and enhancement values, in $\mathrm{dB} \mathrm{HL}$, for overall frequency as a function of CAS duration. The vertical bars represent standard deviations. Suppression value is defined as the difference between pre-acoustic stimulation amplitude and the amplitude during acoustic stimulation. Enhancement value is defined as the difference between pre-acoustic stimulation amplitude and the amplitude after acoustic stimulation. The initial suppression value was significantly higher than suppression values at 5,10 , and 15 minutes $(p<0.05)$. TEOAE: transient evoked otoacoustic emission, CAS: contralateral acoustic stimulatation.

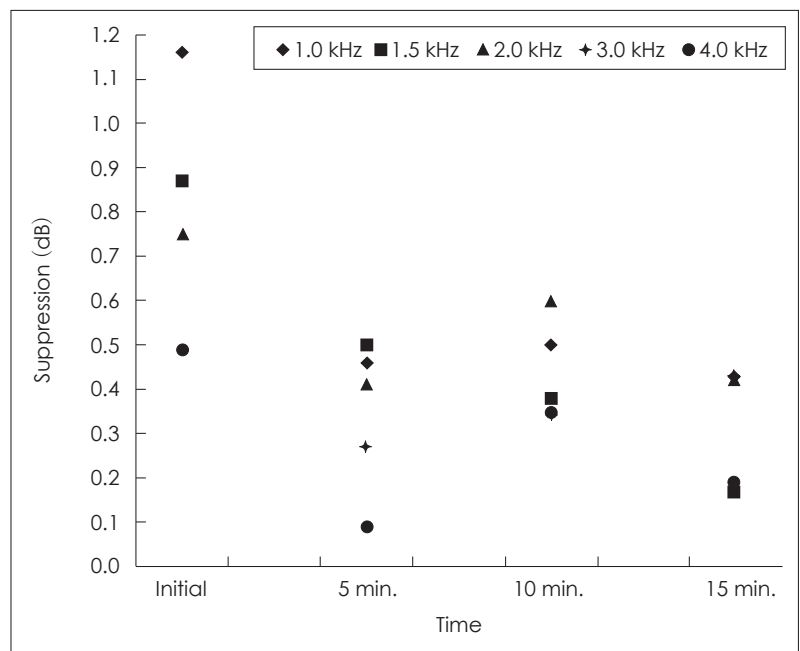

Fig. 4. Difference in TEOAE amplitude suppression among frequency bands during contralateral acoustic stimulation. No significant difference in TEOAE supression was found among the frequencies. TEOAE: transient evoked otoacoustic emission, CAS: contralateral acoustic stimulatation.

and $0.33 \mathrm{~dB}(5.56 \%$ suppression) at 15 minutes during CAS. The initial suppression value was significantly larger than suppression values at 5, 10, and 15 minutes $(p<0.05$ )(Fig. 3). After CAS, the enhancement values in the mean amplitudes for overall frequency were 0.47 (7.93\% enhancement from preacoustic stimulation) at 1 minute, 0.56 (9.44\% enhancement) at 3 minutes, and 0.20 (3.37\% enhancement) at 5 minutes. There were no significant differences among the three values after CAS (Fig. 3).

As frequency increased, a tendency for suppression to decrease was observed during the whole duration with acoustic stimulation; however, among the frequencies, no statistical difference was found with regard to suppression (Fig. 4).

\section{Discussion}

In the present study, we investigated the inhibitory effect of the medial olivocochlear system as measured by TEOAE in the presence of prolonged CAS in adults with normal hearing. Though the maintenance periods of inhibitory effects with CAS differed according to frequency, the amplitude of TEOAEs decreased during a certain period (from the immediate period to 10 minutes, depending on the frequency) before recovering. The TEOAE suppression value for overall frequency showed the highest score at the initial recording and fell abruptly after that point. These results are somewhat different to those of van Zyl, et al.,") in which amplitude suppressions of TEOAE were maintained at all frequencies during 16 minutes of acoustic stimulation and the overall TEOAE suppression values demonstrated no change between recording intervals during CAS. We consider that the present result showed efferent fatigue or sensory adaptation in the medial olivocochlear system. A human study published in 1997 reported that suppression of TEOAE remained constant throughout the entire period of CAS (from 10 to 180 seconds) and that there was no manifestation of significant efferent fatigue. ${ }^{6}$ A study that used distortion product OAE reported that maximal suppression occurred within 2.6 seconds after acoustic stimulation and that the medial olivocochlear effect was maintained for a duration of acoustic stimulation of 20 minutes. ${ }^{7)}$ In contrast, a human study using spontaneous OAE reported efferent fatigue or sensory adaptation, in agreement with the findings of the present study, and reported a rapid reduction in amplitude at the onset of acoustic stimulation followed by a lessening of this reduction with 20 seconds of CAS. ${ }^{8)}$ To evaluate the efferent effect of the medial olivocochlear system, compound action potential (CAP) and ensemble background activity (EBA) have been used in animal studies. One study monitored cochlear CAP during 12 seconds of contralateral broadband sound in anesthetized cats and found that suppression reached a constant steady state 2 seconds after the onset of contralateral sound, followed by a constant state during contralateral sound. When the sound was turned off, suppression disappeared in less than 0.62 seconds. ${ }^{10)}$ In another study using electrical stimulation instead of acoustic stimulation, CAP amplitude was reduced to less than $50 \%$ of control values within $100 \mathrm{~ms}$ after the onset of electrical stimulation, after which CAP amplitude continued to decline slowly by a further $20 \%$ within 1 minute after the onset of stimulation, and the decreased CAP amplitude remained until electrical stimulation ceased. ${ }^{11)}$ Sridhar, et al. ${ }^{11)}$ analyzed the duration properties of the medial olivocochlear system reflex and identified two different effects: the fast effect (rapid 
decrease in amplitude after the onset of stimulation) and the slow effect (further decrease in amplitude after the fast effect). These effects have been measured previously in an animal study using EBA and CAS; the authors reported a rapid $75 \%$ decrease in the total decreased EBA amplitude within $10 \mathrm{~ms}$ after the onset of CAS (fast effect), and an additional slower decrease of $25 \%$ (slow effect) after the fast decrease remained constant during presentation of more than 2 hours of acoustic stimulation. Previous studies, however, were unable to fully explain the mechanism behind these two effects, and no studies have been performed that confirm these effects in humans.

In the present study, we detected a pattern of a longer time period of suppression at lower frequencies: at 1 and $1.5 \mathrm{kHz}$, OAE amplitude reduction remained until recordings at 10 and 5 minutes, respectively, with acoustic stimulation; whereas at 2,3 , and $4 \mathrm{kHz}$, amplitude reduction was present only at the immediate recording with acoustic stimulation. These results imply that the protective mechanism of the medial olivocochlear system is more active at low frequencies than at high frequencies, and may be correlated with the greater damage caused by noise in the high-frequency area in the cochlea. The tendency for greater suppression during acoustic stimulation at lower frequencies is in agreement with this assumption, and was also observed in the study of van Zyl, et al., ${ }^{9)}$ who investigated 16-minute CAS in 10 adults with normal hearing.

At overall 1-4 kHz mean frequency, the maximal suppression value in the current study was $0.76 \mathrm{~dB}$ (approximately $12 \%$ suppression compared with pre-acoustic stimulation), which is less than the maximal suppression value of $>1 \mathrm{~dB}$ reported in the study of van $\mathrm{Zyl}$, et al. ${ }^{9)}$ In the present study, the participants ranged in age from 23 to 35 years with hearing thresholds of less than $20 \mathrm{~dB}$ between 250 and $8000 \mathrm{~Hz}$; in contrast, in the study of van Zyl, et al. the participants ranged in age from 20 to 26 years with hearing thresholds of less than $10 \mathrm{~dB} H \mathrm{HL}$ in the same frequency range. In this latter study, the subjects may have had largely intact cochlear hair cells even at high frequency and extremely good function of the efferent nervous system. The amplitude of TEOAE and efferent suppression with CAS can deteriorate with age and hearing sensitivity. ${ }^{12)}$ Indeed, the pre-acoustic stimulation amplitude of TEOAE was lower in the present study. This observation, combined with the smaller suppression value, may explain why earlier efferent fatigue or sensory adaptation was detected exclusively in the present study.

In recordings obtained after termination of acoustic stimulation, TEOAE amplitude exceeded pre-acoustic amplitudes at $1 \mathrm{kHz}(1,3$, and 5 minutes post-acoustic stimulation), 1.5 $\mathrm{kHz}$ ( 1 and 3 minutes post-acoustic stimulation), and $2 \mathrm{kHz}$
(1 minute and 3 minutes post-acoustic stimulation), showing overshoots. The overshoots at 1.5 and $2 \mathrm{kHz}$ recovered at 5 minutes. Though at $3 \mathrm{kHz}$ and $4 \mathrm{kHz}$, the overshooting patterns were also observed after termination of acoustic stimulation, there were not significant amplitude increases. It might be caused by more sensitive medial olivocochlear system at low frequencies as a longer time period of suppression at lower frequencies in the presence of CAS. In the study of van Zyl, et al., ${ }^{9)}$ overshoot of TEOAE amplitude appeared at not 1 minute but 3 minutes after termination of acoustic stimulation; however, the time course was not studied for a longer duration than 3 minutes, and the authors did not mention the recovery of TEOAE amplitude. A previous study with guinea pigs showed increased sound-evoked vibrations of the basilar membrane, lasting 100-200 seconds after the termination of electrical stimulation. ${ }^{13)}$ In contrast, a different result was obtained in a human study using DPOAE with presentation of 20 minutes of CAS. ${ }^{7)}$ The authors reported that DPOAE amplitude remained suppressed at the recording obtained 1 minute after termination of acoustic stimulation, and that the suppression recovered in subsequent recordings at 1-minute intervals, indicating residual suppression after the termination of acoustic sound. They attributed this phenomenon to the medial olivocochlear slow effect or to the persistence of the efferent effect after the termination of acoustic sound.

Even though we used a similar experimental setup to that of van Zyl, et al., we found several different phenomena: efferent fatigue during CAS, and overshooting and recovery at several frequencies after CAS. Consequently, more research is necessary to fully understand the underlying mechanisms of the suppressive effect.

The limitation of the current study is the absence of the control group whose TEOAEs were checked at the same time interval without CAS.

\section{Conclusion}

We revealed significant TEOAE amplitude reduction with CAS in adults with normal hearing, and verified the suppressive effect of the medial olivocochlear system. The present results demonstrate that TEOAE suppression occurs immediately after CAS, and indicate the possible manifestation of efferent fatigue or sensory adaptation. After the termination of acoustic stimulation, transient TEOAE amplitude enhancement (overshooting) and spontaneous recovery were observed.

\section{REFERENCES}

1) Kemp DT. Stimulated acoustic emissions from within the human 
auditory system. J Acoust Soc Am 1978;64:1386-91.

2) Brown MC, de Venecia RK, Guinan JJ Jr. Responses of medial olivocochlear neurons. Specifying the central pathways of the medial olivocochlear reflex. Exp Brain Res 2003;153:491-8.

3) Fitch RH, Read H, Benasich AA. Neurophysiology of speech perception in normal and impaired systems. In: Jahn AF, Santos-Sacchi J, editors. Physiology of the ear. 2nd ed. San diego: Singular Publishing Groups, Inc.;2001. p.639-50.

4) Reiter ER, Liberman MC. Efferent-mediated protection from acoustic overexposure: relation to slow effects of olivocochlear stimulation. J Neurophysiol 1995;73:506-14.

5) Puel JL, Bonfils P, Pujol R. Selective attention modifies the active micromechanical properties of the cochlea. Brain Res 1988;447:380-3.

6) Giraud AL, Collet L, Chéry-Croze S. Suppression of otoacoustic emission is unchanged after several minutes of contralateral acoustic stimulation. Hear Res 1997;109:78-82.

7) Moulin A, Carrier S. Time course of the medial olivocochlear efferent effect on otoacoustic emissions in humans. Neuroreport 1998;9: $3741-4$
8) Zhao W, Dhar S. The effect of contralateral acoustic stimulation on spontaneous otoacoustic emissions. J Assoc Res Otolaryngol 2010; 11:53-67.

9) van Zyl A, Swanepoel D, Hall JW 3rd. Effect of prolonged contralateral acoustic stimulation on transient evoked otoacoustic emissions. Hear Res 2009;254:77-81.

10) Puria S, Guinan JJ Jr, Liberman MC. Olivocochlear reflex assays: effects of contralateral sound on compound action potentials versus ear-canal distortion products. J Acoust Soc Am 1996;99:500-7.

11) Sridhar TS, Liberman MC, Brown MC, Sewell WF. A novel cholinergic "slow effect" of efferent stimulation on cochlear potentials in the guinea pig. J Neurosci 1995;15(5 Pt 1):3667-78.

12) Keppler H, Dhooge I, Corthals P, Maes L, D'haenens W, Bockstael A, et al. The effects of aging on evoked otoacoustic emissions and efferent suppression of transient evoked otoacoustic emissions. Clin Neurophysiol 2010;121:359-65.

13) Cooper NP, Guinan JJ Jr. Separate mechanical processes underlie fast and slow effects of medial olivocochlear efferent activity. J Physiol 2003;548(Pt 1):307-12. 